\title{
Fractional integral operators on Herz spaces for supercritical indices
}

\author{
Yasuo Komori-Furuya
}

(Communicated by Hans Triebel)

2000 Mathematics Subject Classification. 42B20.

Keywords and phrases. Herz space, fractional integral, BMO, Lipschitz space.

Abstract. We consider the boundedness of fractional integral operators $I_{\beta}$ on Herz spaces $K_{q}^{\alpha, p}\left(R^{n}\right)$, where $q \geq n / \beta$. We introduce a new function space that is a variant of Lipschitz space. Our results are optimal.

\section{Introduction}

Since Beurling [1] introduced the Beurling algebras and Herz [5] generalized these spaces, many studies have been done for Herz spaces (see, for example, [3], [4] and [9]). Li and Yang [7] considered the boundedness of fractional integral operators on Herz spaces. In this paper we consider the boundedness of fractional integral operators on Herz spaces for supercritical indices and we shall show our results are optimal.

The following notation is used: For a set $E \subset \mathbb{R}^{n}$ we denote the Lebesgue measure of $E$ by $|E|$. We denote the characteristic function of $E$ by $\chi_{E}$. We write a ball of radius $R$ centered at $x_{0}$ by $B\left(x_{0}, R\right)=\left\{x ;\left|x-x_{0}\right|<R\right\}$, and write $A_{k}=\left\{x \in \mathbb{R}^{n} ; 2^{k-1}<|x| \leq 2^{k}\right\}$ where $k \in \mathbb{Z}$. 
First we define homogeneous Herz spaces and fractional integral. Let $0<p<\infty, 1 \leq q<\infty$ and $\alpha \in \mathbb{R}^{1}$.

Definition 1. $\dot{K}_{q}^{\alpha, p}\left(\mathbb{R}^{n}\right)=\left\{f \in L_{\mathrm{loc}}^{q}\left(\mathbb{R}^{n} \backslash\{0\}\right) ;\|f\|_{\dot{K}_{q}^{\alpha, p}}<\infty\right\}$, where

$$
\|f\|_{\dot{K}_{q}^{\alpha, p}}=\left\{\sum_{k=-\infty}^{\infty} 2^{k \alpha p}\left(\int_{A_{k}}|f(x)|^{q} d x\right)^{p / q}\right\}^{1 / p} .
$$

Remark . $K_{q}^{0, q}\left(\mathbb{R}^{n}\right)=L^{q}\left(\mathbb{R}^{n}\right)$.

\section{Definition 2.}

$$
I_{\beta} f(x)=\int_{\mathbb{R}^{n}} \frac{1}{|x-y|^{n-\beta}} f(y) d y \text { for } \quad 0<\beta<n .
$$

The next proposition is well-known (see, for example, [14]).

Proposition 1. $I_{\beta}$ is bounded from $L^{q_{1}}\left(\mathbb{R}^{n}\right)$ to $L^{q_{2}}\left(\mathbb{R}^{n}\right)$ where $1 / q_{2}=1 / q_{1}-\beta / n>0$.

Li and Yang [7] proved the following.

Proposition 2. $I_{\beta}$ is bounded from $\dot{K}_{q_{1}}^{\alpha, p}\left(\mathbb{R}^{n}\right)$ to $\dot{K}_{q_{2}}^{\alpha, p}\left(\mathbb{R}^{n}\right)$ where

(1) $1 / q_{2}=1 / q_{1}-\beta / n>0, \beta-n / q_{1}<\alpha<n\left(1-1 / q_{1}\right)$ and $0<p<\infty$.

When $q_{1} \geq n / \beta$, we know the following proposition (see [12] and [14]). We define Lipschitz spaces and modified fractional integral. Let $0 \leq \varepsilon<1$.

\section{Definition 3.}

$$
\operatorname{Lip}_{\varepsilon}\left(\mathbb{R}^{n}\right)=\left\{f ;\|f\|_{L i p_{\varepsilon}}=\sup _{Q} \inf _{c} \frac{1}{|Q|^{1+\varepsilon / n}} \int_{Q}|f(x)-c| d x<\infty\right\},
$$

where the supremum is taken over all balls $Q \subset \mathbb{R}^{n}$.

We denote $\operatorname{Lip}_{0}\left(\mathbb{R}^{n}\right)=B M O\left(\mathbb{R}^{n}\right)$.

Proposition 3. When $0<\varepsilon<1$,

$$
\|f\|_{L i p_{\varepsilon}} \approx \sup _{x \neq y} \frac{|f(x)-f(y)|}{|x-y|^{\varepsilon}} .
$$

\section{Definition 4.}

$$
\tilde{I}_{\beta} f(x)=\int_{\mathbb{R}^{n}}\left\{\frac{1}{|x-y|^{n-\beta}}-\frac{1}{|y|^{n-\beta}} \chi_{\{|y| \geq 1\}}\right\} f(y) d y \quad \text { for } \quad 0<\beta<n .
$$


Proposition 4. $\widetilde{I}_{\beta}$ is bounded from $L^{q}\left(\mathbb{R}^{n}\right)$ to $\operatorname{Lip}_{\beta-n / q}\left(\mathbb{R}^{n}\right)$ where

$$
0 \leq \beta-n / q<1 .
$$

Especially $\widetilde{I}_{\beta}$ is bounded from $L^{n / \beta}\left(\mathbb{R}^{n}\right)$ to $B M O\left(\mathbb{R}^{n}\right)$.

We consider the boudedness of $I_{\beta}$ on $\dot{K}_{q}^{\alpha, p}$ where $q \geq n / \beta$. Lu and Yang [8] (see also [6]) proved the following.

Proposition 5. Assume that $q_{1} \geq n / \beta$ and $0<p<n / \beta$. Then $I_{\beta}$ is bounded from $K_{q_{1}}^{\alpha_{1}, p}\left(\mathbb{R}^{n}\right)$ to $K_{q_{2}}^{\alpha_{2}, p}\left(\mathbb{R}^{n}\right)$ for appropriate $\alpha_{1}, \alpha_{2}$ and $q_{2}$.

However this result does not imply Proposition 4, because Proposition 5 is not applicable to the case $p=q_{1}$. Note that $K_{q}^{0, q}=L^{q}$. In this paper we consider the theorem that is an extension of Proposition 4. By a simple observation we obtain the following result. We define $C M O$ (central mean oscillation) introduced by García-Cuerva [3]. Let $0 \leq \varepsilon<1$.

\section{Definition 5.}

$C M O_{\varepsilon}\left(\mathbb{R}^{n}\right)=\left\{f ;\|f\|_{C M O_{\varepsilon}}=\sup _{R>0} \inf _{c} \frac{1}{|B(0, R)|^{1+\varepsilon / n}} \int_{B(0, R)}|f(x)-c| d x<\infty\right\}$.

Theorem 1. $\widetilde{I}_{\beta}$ is bounded from $\dot{K}_{q}^{\alpha, p}\left(\mathbb{R}^{n}\right)$ to $C M O_{\beta-n / q-\alpha}\left(\mathbb{R}^{n}\right)$ where

$$
q \geq n / \beta, \beta-n / q-1<\alpha \leq \beta-n / q \text { and } 0<p<\infty .
$$

The spaces $C M O_{\beta-n / q-\alpha}$ are independent of $p$, but the operator norms $\left\|\widetilde{I}_{\beta}\right\|_{\dot{K}_{q}^{\alpha, p} \rightarrow C M O_{\beta-n / q-\alpha}}$ depend on $p$.

Note that the modified fractional integrals $\widetilde{I}_{\beta}$ are well-defined on $\dot{K}_{q}^{\alpha, p}$ where $\beta-n / q-1<\alpha$. Compare with the condition (1).

However this result is insufficient, because when $\alpha=0$ and $p=q=n / \beta$ it says that

$$
\widetilde{I}_{\beta}: L^{n / \beta} \rightarrow C M O_{0} .
$$

Since $B M O \subsetneq C M O_{0}$, it does not imply Proposition 4 .

In the following, we consider the theorem that is consistent with Proposition 4 when $\alpha=0$ and $p=q$.

In Section 4 , we shall show that $\widetilde{I}_{\beta}$ is not bounded from $K_{q}^{\alpha, p}$ to $\mathrm{Lip}_{\beta-n / q}$ in general, therefore we need to consider other function spaces. 


\section{Main Theorem}

For our purpose we consider a variant of Lipschitz space. Our definition is the following. Let $0 \leq \varepsilon<1$ and $\lambda \in \mathbb{R}^{1}$.

\section{Definition 6.}

$$
\begin{aligned}
& \operatorname{Lip}_{\varepsilon}^{\lambda}\left(\mathbb{R}^{n}\right) \\
& =\left\{f ;\|f\|_{L i p_{\varepsilon}^{\lambda}}=\sup _{\substack{x \in \mathbb{R}^{n} \\
R>0}} \inf _{c} \frac{1}{(|x|+R)^{\lambda}} \frac{1}{|B(x, R)|^{1+\varepsilon / n}} \int_{B(x, R)}|f(y)-c| d y<\infty\right\} .
\end{aligned}
$$

Remarks . $\operatorname{Lip} p_{\varepsilon}^{0}\left(\mathbb{R}^{n}\right)=\operatorname{Lip}_{\varepsilon}\left(\mathbb{R}^{n}\right)$ and $\operatorname{Lip} p_{0}^{0}\left(\mathbb{R}^{n}\right)=B M O\left(\mathbb{R}^{n}\right)$.

$$
\|f\|_{L i p_{\varepsilon}^{\lambda}} \approx \sup _{\substack{x \in \mathbb{R}^{n} \\ R>0}} \frac{1}{(|x|+R)^{\lambda}} \frac{1}{|B(x, R)|^{1+\varepsilon / n}} \int_{B(x, R)}\left|f(y)-f_{B(x, R)}\right| d y<\infty,
$$

where $f_{B(x, R)}=\frac{1}{|B(x, R)|} \int_{B(x, R)} f(y) d y$.

Such function spaces are introduced by Nakai et al. [10], [11], [2]. They consider more general function spaces, that is, generalized Campanato spaces:

$$
\begin{aligned}
& \operatorname{Lip}_{\varepsilon}^{\varphi}\left(\mathbb{R}^{n}\right) \\
& =\left\{f ;\|f\|_{L i p_{\varepsilon}^{\varphi}}=\sup _{\substack{x \in \mathbb{R}^{n} \\
R>0}} \inf _{c} \frac{1}{\varphi(x, R)} \frac{1}{|B(x, R)|^{1+\varepsilon / n}} \int_{B(x, R)}|f(y)-c| d y<\infty\right\} .
\end{aligned}
$$

Before we state our results, we investigate some properties of $\operatorname{Lip}_{\varepsilon}^{\lambda}\left(\mathbb{R}^{n}\right)$. Since $\operatorname{Lip}_{\varepsilon}^{\beta-n / q-\alpha-\varepsilon} \subset C M O_{\beta-n / q-\alpha}$, Theorem 1 is obtained from Theorems 2 and 3 below. The following property is easily obtained from the definition.

$$
\operatorname{Lip}_{\varepsilon^{\prime}}^{\lambda^{\prime}}\left(\mathbb{R}^{n}\right) \subset \operatorname{Lip} p_{\varepsilon}^{\lambda}\left(\mathbb{R}^{n}\right) \quad \text { where } \quad \varepsilon^{\prime}-\varepsilon=\lambda-\lambda^{\prime} \geq 0 .
$$

Especially

$$
\begin{array}{lll}
\operatorname{Lip}_{\beta-n / q}^{-\alpha}\left(\mathbb{R}^{n}\right) \subset \operatorname{Lip}_{\beta-n / q-\alpha}\left(\mathbb{R}^{n}\right) & \text { if } & \alpha>0, \\
\operatorname{Lip}_{\beta-n / q-\alpha}\left(\mathbb{R}^{n}\right) \subset \operatorname{Lip}_{\beta-n / q}^{-\alpha}\left(\mathbb{R}^{n}\right) & \text { if } & \alpha<0 .
\end{array}
$$

This observation is important for our theorems (see Remark below). We give some examples. 


$$
\begin{aligned}
& |x|^{1+\varepsilon} \in \operatorname{Lip}_{\varepsilon}^{1}\left(\mathbb{R}^{n}\right), \quad|x|^{-\varepsilon} \in \operatorname{Lip}_{0}^{-\varepsilon}\left(\mathbb{R}^{n}\right) \quad \text { where } 0 \leq \varepsilon<1 \\
& (\log |x|)^{2} \chi_{\{|x| \geq 1\}} \in \operatorname{Lip} p_{0}^{1}\left(\mathbb{R}^{n}\right) \backslash B M O\left(\mathbb{R}^{n}\right), \\
& (\log |x|)^{2} \chi_{\{|x| \leq 1\}} \in \operatorname{Lip}_{0}^{-1}\left(\mathbb{R}^{n}\right) \backslash B M O\left(\mathbb{R}^{n}\right) .
\end{aligned}
$$

Our main result is the following.

Theorem 2. Let $q \geq n / \beta, 0<p<\infty$ and $\beta-n / q-1<\alpha<n-n / q$. If $0 \leq \beta-n / q<1+\min (0, \alpha)$, then $\widetilde{I}_{\beta}$ is bounded from $\dot{K}_{q}^{\alpha, p}\left(\mathbb{R}^{n}\right)$ to $\operatorname{Lip}_{\beta-n / q}^{-\alpha}\left(\mathbb{R}^{n}\right)$.

Remark. The spaces $L i p_{\beta-n / q}^{-\alpha}$ are defined only for $\beta-n / q<1$. We shall show that the index $\beta-n / q$ is optimal in Section 4 , and also show that if $\alpha<0$ then $\widetilde{I}_{\beta}$ is not bounded from $\dot{K}_{q}^{\alpha, p}$ to $\operatorname{Lip}_{\beta-n / q-\alpha}$ (see (5)).

When $\alpha>0$ and $1 \leq \beta-n / q<1+\alpha$, we have the following result.

Theorem 3. Let $q \geq n / \beta$ and $0<p<\infty$. If $\alpha \geq 0$ and $0 \leq$ $\beta-n / q-\alpha<1$, then $\widetilde{I}_{\beta}$ is bounded from $\dot{K}_{q}^{\alpha, p}\left(\mathbb{R}^{n}\right)$ to $\operatorname{Lip}_{\beta-n / q-\alpha}\left(\mathbb{R}^{n}\right)$.

Remark. The case $\beta-n / q<1$ is contained in Theorem 2 .

\section{Corollary .}

$$
\begin{aligned}
& \widetilde{I}_{\beta}: \dot{K}_{q}^{0, p}\left(\mathbb{R}^{n}\right) \rightarrow \operatorname{Lip} p_{\beta-n / q}\left(\mathbb{R}^{n}\right) \quad \text { if } \quad 0<\beta-n / q<1, \\
& \widetilde{I}_{\beta}: \dot{K}_{n / \beta}^{0, p}\left(\mathbb{R}^{n}\right) \rightarrow B M O\left(\mathbb{R}^{n}\right) .
\end{aligned}
$$

Since $L^{q} \subset K_{q}^{0, p}$ when $q \leq p$, Corollary is an extension of Proposition 4.

\section{Proofs}

In order to prove Theorem 2 we prepare two lemmas.

Lemma 1. Assume that $1 \geq 1 / s>\max (1 / q+\alpha / n, 1 / q)$. Then

$$
\left(\int_{B\left(0,2^{k}\right)}|f(x)|^{s} d x\right)^{1 / s} \leq C 2^{k(n(1 / s-1 / q)-\alpha)}\|f\|_{\dot{K}_{q}^{\alpha, p} .}
$$

Throughout this paper, $C$ is a positive constant which is independent of essential parameters and not necessarily same at each occurrence. 
Proof. We write

$$
\begin{aligned}
& \left(\int_{B\left(0,2^{k}\right)}|f(x)|^{s} d x\right)^{1 / s}=\left(\sum_{j \leq k} \int_{A_{j}}|f(x)|^{s} d x\right)^{1 / s} \\
& \quad \leq C\left\{\sum_{j \leq k} 2^{j(n(1-s / q)-\alpha s)} \cdot 2^{j \alpha s}\left(\int_{A_{j}}|f(x)|^{q} d x\right)^{s / q}\right\}^{1 / s}=: X .
\end{aligned}
$$

If $p>s$. Let $1 / t+s / p=1$. Then we have

$$
\begin{aligned}
X & \leq C\left\{\sum_{j \leq k} 2^{j(n(1-s / q)-\alpha s) t}\right\}^{1 / t s}\left\{\sum_{j \leq k} 2^{j \alpha p}\left(\int_{A_{j}}|f(x)|^{q} d x\right)^{p / q}\right\}^{1 / p} \\
& \leq C 2^{k(n(1 / s-1 / q)-\alpha)}\|f\|_{\dot{K}_{q}^{\alpha, p}}
\end{aligned}
$$

since $n(1 / s-1 / q)-\alpha>0$. If $p \leq s$, then we have

$$
\begin{aligned}
X & \leq\left\{\sum_{j \leq k} 2^{j(n(1-s / q)-\alpha s) p / s} \cdot 2^{j \alpha p}\left(\int_{A_{j}}|f(x)|^{q} d x\right)^{p / q}\right\}^{1 / p} \\
& \leq C 2^{k(n(1 / s-1 / q)-\alpha)}\|f\|_{\dot{K}_{q}^{\alpha, p} .}
\end{aligned}
$$

Lemma 2. Assume that $\beta-n / q-1<\alpha$. Then

(7)

$$
\int_{|y| \geq 2^{k}} \frac{|f(y)|}{|y|^{n+1-\beta}} d y \leq C 2^{k(\beta-n / q-\alpha-1)}\|f\|_{\dot{K}_{q}^{\alpha, p} .}
$$

Proof. We write

$$
\begin{aligned}
& \int_{|y| \geq 2^{k}} \frac{|f(y)|}{|y|^{n+1-\beta}} d y=\sum_{j \geq k} \int_{A_{j}} \frac{|f(y)|}{|y|^{n+1-\beta}} d y \\
& \quad \leq C \sum_{j \geq k} 2^{j(\beta-n / q-\alpha-1)} \cdot 2^{j \alpha}\left(\int_{A_{j}}|f(y)|^{q} d y\right)^{1 / q}=: X .
\end{aligned}
$$

If $p>1$, then we have

$$
\begin{aligned}
X & \leq C\left\{\sum_{j \geq k} 2^{j(\beta-n / q-\alpha-1) p^{\prime}}\right\}^{1 / p^{\prime}}\left\{\sum_{j \geq k} 2^{j \alpha p}\left(\int_{A_{j}}|f(y)|^{q} d y\right)^{p / q}\right\}^{1 / p} \\
& \leq C 2^{k(\beta-n / q-\alpha-1)}\|f\|_{\dot{K}_{q}^{\alpha, p}}
\end{aligned}
$$

since $\beta-n / q-\alpha-1<0$. 
If $p \leq 1$, then we have

$$
\begin{aligned}
X & \leq C\left\{\sum_{j \geq k} 2^{j(\beta-n / q-\alpha-1) p} \cdot 2^{j \alpha p}\left(\int_{A_{j}}|f(y)|^{q} d y\right)^{p / q}\right\}^{1 / p} \\
& \leq C 2^{k(\beta-n / q-\alpha-1)}\|f\|_{\dot{K}_{q}^{\alpha, p} .}
\end{aligned}
$$

Now we are in a position to prove Theorem 2 .

Proof. [Proof of Theorem 2.] Let $\varepsilon=\beta-n / q$. Fix a ball $Q=B\left(x_{0}, R\right)$ and we estimate

$$
\inf _{c} \frac{\left(\left|x_{0}\right|+R\right)^{\alpha}}{|Q|^{1+\varepsilon / n}} \int_{Q}\left|\widetilde{I}_{\beta} f(x)-c\right| d x .
$$

Let $k$ be the least integer such that $Q \subset B\left(0,2^{k}\right)$. Note that

$$
\left|x_{0}\right|+R \approx 2^{k} .
$$

We consider three cases:

(i) $Q \cap B\left(0,2^{k-2}\right) \neq \emptyset$,

(ii) $Q \cap B\left(0,2^{k-2}\right)=\emptyset$ and $R \geq 2^{k-4}$,

(iii) $Q \cap B\left(0,2^{k-2}\right)=\emptyset$ and $R<2^{k-4}$.

The case (i) or (ii). Note that $|Q| \geq C 2^{k n}$ in both cases. We write

$$
f(x)=f(x) \chi_{B\left(0,2^{k+1}\right)}+f(x) \chi_{\mathrm{C}_{B}\left(0,2^{k+1}\right)}=: f_{1}(x)+f_{2}(x) .
$$

First we estimate $\widetilde{I}_{\beta} f_{1}$. Let $c_{1}=-\int_{|y| \geq 1} f_{1}(y) /|y|^{n-\beta} d y$. Then $\widetilde{I}_{\beta} f_{1}(x)-c_{1}=I_{\beta} f_{1}(x)$. Applying Lemma 1 for $s=1$, we have

$$
\begin{aligned}
\int_{Q}\left|I_{\beta} f_{1}(x)\right| d x & \leq \int_{B\left(0,2^{k+1}\right)}|f(y)|\left(\int_{Q} \frac{1}{|x-y|^{n-\beta}} d x\right) d y \\
& \leq C 2^{k(n(1-1 / q)-\alpha)}|Q|^{\beta / n}\|f\|_{\dot{K}_{q}^{\alpha, p}}
\end{aligned}
$$

and obtain

$$
\frac{1}{|Q|^{1+\varepsilon / n}} \int_{Q}\left|\widetilde{I}_{\beta} f_{1}-c_{1}\right| d x \leq C\left(\left|x_{0}\right|+R\right)^{-\alpha}\|f\|_{\dot{K}_{q}^{\alpha, p}}
$$


Next we estimate $\widetilde{I}_{\beta} f_{2}$. Let $c_{2}=\widetilde{I}_{\beta} f\left(x_{0}\right)$. For any $x \in Q$,

$$
\begin{aligned}
\left|\widetilde{I}_{\beta} f_{2}(x)-c_{2}\right| & \leq \int\left|\frac{1}{|x-y|^{n-\beta}}-\frac{1}{\left|x_{0}-y\right|^{n-\beta}}\right|\left|f_{2}(y)\right| d y \\
& \leq C R \int_{|y| \geq 2^{k+1}} \frac{|f(y)|}{\left|x_{0}-y\right|^{n+1-\beta}} d y \\
& \leq C R 2^{k(\beta-n / q-\alpha-1)}\|f\|_{\dot{K}_{q}^{\alpha, p}}
\end{aligned}
$$

by Lemma 2 , and we have

$$
\frac{1}{|Q|^{1+\varepsilon / n}} \int_{Q}\left|\widetilde{I}_{\beta} f_{2}-c_{2}\right| d x \leq C\left(\left|x_{0}\right|+R\right)^{-\alpha}\|f\|_{\dot{K}_{q}^{\alpha, p}}
$$

The case (iii). We write

$$
\begin{aligned}
f(x) & =f(x) \chi_{B\left(x_{0}, 2 R\right)}+f(x) \chi_{\mathrm{C}\left\{2^{k-3}<|x| \leq 2^{k+1}\right\}}+f(x) \chi_{\left\{2^{k-3}<|x| \leq 2^{k+1}\right\} \backslash B\left(x_{0}, 2 R\right)} \\
& =: f_{1}(x)+f_{2}(x)+f_{3}(x) .
\end{aligned}
$$

First we estimate $\widetilde{I}_{\beta} f_{1}$. Let $c_{1}=-\int_{|y| \geq 1} f_{1}(y) /|y|^{n-\beta} d y$. By the same estimate as (9), we have

$$
\begin{aligned}
\int_{Q}\left|\widetilde{I}_{\beta} f_{1}(x)-c_{1}\right| d x & \leq C|Q|^{\beta / n} \int_{B\left(x_{0}, 2 R\right)}|f(y)| d y \\
& \leq C\left(\int_{B\left(x_{0}, 2 R\right)}|f(y)|^{q} d y\right)^{1 / q}|Q|^{1-1 / q+\beta / n} \\
& \leq C 2^{-k \alpha}|Q|^{1-1 / q+\beta / n}\|f\|_{\dot{K}_{q}^{\alpha, p}}
\end{aligned}
$$

because $B\left(x_{0}, 2 R\right) \subset \cup_{j=k-2}^{k+1} A_{j}$. Therefore we obtain

$$
\frac{1}{|Q|^{1+\varepsilon / n}} \int_{Q}\left|I_{\beta} f_{1}(x)-c_{1}\right| d x \leq C\left(\left|x_{0}\right|+R\right)^{-\alpha}\|f\|_{\dot{K}_{q}^{\alpha, p}}
$$

Next we estimate $\widetilde{I}_{\beta} f_{2}$. Let $c_{2}=\widetilde{I}_{\beta} f_{2}\left(x_{0}\right)$. By the same estimate as (10) and Lemma 1 for $s=1$, it follows that for any $x \in Q$,

$$
\begin{aligned}
\left|\widetilde{I}_{\beta} f_{2}(x)-c_{2}\right| & \leq C R\left(\int_{|y| \geq 2^{k+1}} \frac{|f(y)|}{\left|x_{0}-y\right|^{n+1-\beta}} d y+\int_{|y| \leq 2^{k-3}} \frac{|f(y)|}{\left|x_{0}-y\right|^{n+1-\beta}} d y\right) \\
& \leq C R\left(2^{k(\beta-n / q-\alpha-1)}\|f\|_{\dot{K}_{q}^{\alpha, p}}+2^{-k(n+1-\beta)} \int_{|y| \leq 2^{k-3}}|f(y)| d y\right) \\
& \leq C R\left(2^{k(\beta-n / q-\alpha-1)}\|f\|_{\dot{K}_{q}^{\alpha, p}}+2^{-k(n+1-\beta)} \cdot 2^{k(n-n / q-\alpha)}\|f\|_{\dot{K}_{q}^{\alpha, p}}\right) \\
& \leq C R 2^{k(\beta-n / q-\alpha-1)}\|f\|_{\dot{K}_{q}^{\alpha, p}}
\end{aligned}
$$


Since $\beta-n / q-1<0$, we obtain

$$
\frac{1}{|Q|^{1+\varepsilon / n}} \int_{Q}\left|I_{\beta} f_{2}(x)-c_{2}\right| d x \leq C\left(\left|x_{0}\right|+R\right)^{-\alpha}\|f\|_{\dot{K}_{q}^{\alpha, p}} .
$$

Finally we estimate $\widetilde{I}_{\beta} f_{3}$. Let $c_{3}=\widetilde{I}_{\beta} f_{3}\left(x_{0}\right)$. By the same estimate as $f_{2}$, it follows that for any $x \in Q$,

$$
\begin{aligned}
\left|\widetilde{I}_{\beta} f_{3}(x)-c_{3}\right| & \leq C R \int_{\left|x_{0}-y\right|>2 R} \frac{\left|f_{3}(y)\right|}{\left|x_{0}-y\right|^{n+1-\beta}} d y \\
& \leq C R\left(\int_{\left|x_{0}-y\right|>2 R} \frac{d y}{\left|x_{0}-y\right|^{(n+1-\beta) q^{\prime}}}\right)^{1 / q^{\prime}}\left(\int_{2^{k-3}<|y| \leq 2^{k+1}}|f(y)|^{q} d y\right)^{1 / q} \\
& \leq C R \cdot R^{-n-1+\beta+n / q^{\prime}} 2^{-k \alpha}\|f\|_{\dot{K}_{q}^{\alpha, p}} \\
& \leq C R^{\beta-n / q} 2^{-k \alpha}\|f\|_{\dot{K}_{q}^{\alpha, p}},
\end{aligned}
$$

and we obtain

$$
\frac{1}{|Q|^{1+\varepsilon / n}} \int_{Q}\left|I_{\beta} f_{3}(x)-c_{2}\right| d x \leq C\left(\left|x_{0}\right|+R\right)^{-\alpha}\|f\|_{\dot{K}_{q}^{\alpha, p}}
$$

The following result is known, see e.g., [2], [11] and [13].

\section{Proposition 6.}

$\left\|\widetilde{I}_{\beta} f\right\|_{L_{i p_{\beta-n / t}}} \leq C\|f\|_{M_{s}^{t}}$ where $0 \leq \beta-n / t<1$ and $1<s \leq t$.

Also we need the following

\section{Definition 7.}

$$
M_{s}^{t}\left(\mathbb{R}^{n}\right)=\left\{f ;\|f\|_{M_{s}^{t}}=\sup _{Q} \frac{1}{|Q|^{1 / s-1 / t}}\left(\int_{Q}|f(x)|^{s} d x\right)^{1 / s}<\infty\right\},
$$

where the supremum is taken over all balls $Q \subset \mathbb{R}^{n}$.

Now, we prove Theorem 3.

Proof. [Proof of Theorem 3.] It suffices to show that for $\alpha \geq 0,1 / t=$ $1 / q+\alpha / n$ and $1<s<t$,

$$
K_{q}^{\alpha, p}\left(\mathbb{R}^{n}\right) \subset M_{s}^{t}\left(\mathbb{R}^{n}\right)
$$

since then by Proposition 6 we obtain

$$
\left\|\widetilde{I}_{\beta} f\right\|_{L i p_{\beta-n / q-\alpha}}=\left\|\widetilde{I}_{\beta} f\right\|_{L i p_{\beta-n} / t} \leq C\|f\|_{M_{s}^{t}} \leq C\|f\|_{K_{q}^{\alpha, p}} .
$$


For any ball $Q$, let $k$ be the least integer such that $Q \subset B\left(0,2^{k}\right)$.

First we consider the case $Q \cap B\left(0,2^{k-2}\right) \neq \emptyset$. Note that $|Q| \geq C 2^{k n}$. By Lemma 1, we have

$$
\frac{1}{|Q|^{1 / s-1 / t}}\left(\int_{Q}|f(x)|^{s} d x\right)^{1 / s} \leq C \frac{2^{k(n / s-n / q-\alpha)}}{|Q|^{1 / s-1 / t}}\|f\|_{K_{q}^{\alpha, p}} \leq C\|f\|_{K_{q}^{\alpha, p}}
$$

Next we consider the case $Q \cap B\left(0,2^{k-2}\right)=\emptyset$. Note that $Q \subset$ $A_{k-1} \cup A_{k}$. By Hörder's inequality we have

$$
\begin{aligned}
& \frac{1}{|Q|^{1 / s-1 / t}}\left(\int_{Q}|f(x)|^{s} d x\right)^{1 / s} \leq|Q|^{1 / t-1 / q}\left(\int_{Q}|f(x)|^{q} d x\right)^{1 / q} \\
\leq & C 2^{k \alpha}\left(\int_{A_{k-1}}|f(x)|^{q} d x+\int_{A_{k}}|f(x)|^{q} d x\right)^{1 / q} \leq C\|f\|_{K_{q}^{\alpha, p} .}
\end{aligned}
$$

By (11) and (12), we obtain the desired result.

\section{Counterexample}

We show that $\widetilde{I}_{\beta}$ is not bounded from $K_{q}^{\alpha, p}\left(\mathbb{R}^{n}\right)$ to $\operatorname{Lip}_{\beta-n / q}\left(\mathbb{R}^{n}\right)$ when $\alpha \neq 0$.

Proposition 7. If $\widetilde{I}_{\beta}$ is bounded from $K_{q}^{\alpha, p}\left(\mathbb{R}^{n}\right)$ to $\operatorname{Lip} \bar{\varepsilon}^{\lambda}\left(\mathbb{R}^{n}\right)$, then $\varepsilon+\lambda=\beta-n / q-\alpha$.

Proof. We denote $f_{t}(x)=f(x / t)$. Then $\left\|f_{t}\right\|_{L i p_{\varepsilon}^{\lambda}} t^{-\varepsilon-\lambda}\|f\|_{L i p_{\varepsilon}^{\lambda}}$ and $I_{\beta} f_{t}(x)=t^{\beta}\left(I_{\beta} f\right)(x / t)$. Let $f_{0}(x)=\chi_{\{1<|x|<2\}}$ and $\tilde{f}_{j}(x)=$ $2^{-j(\alpha+n / q)} f_{0}\left(x / 2^{j}\right)$. Then $\left\|\widetilde{f}_{j}\right\|_{K_{q}^{\alpha, p}}=C$. On the other hand we have

$$
\begin{aligned}
\left\|\widetilde{I}_{\beta} \widetilde{f}_{j}\right\|_{L i p_{\varepsilon}^{\lambda}} & =\left\|I_{\beta} \widetilde{f}_{j}\right\|_{L i p_{\varepsilon}^{\lambda}}=2^{-j(\alpha+n / q)} 2^{j \beta} 2^{-j(\varepsilon+\lambda)}\left\|I_{\beta} f_{0}\right\|_{L i p_{\varepsilon}^{\lambda}} \\
& =2^{j(\beta-\alpha-n / q-\varepsilon-\lambda)}\left\|I_{\beta} f_{0}\right\|_{L i p_{\varepsilon}^{\lambda}} .
\end{aligned}
$$

Therefore, if $\sup _{j}\left\|\widetilde{I}_{\beta} \widetilde{f}_{j}\right\|_{L i p_{\varepsilon}^{\lambda}} /\left\|\widetilde{f}_{j}\right\|_{K_{q}^{\alpha, p}}<\infty$, then $\varepsilon+\lambda=\beta-n / q-\alpha$.

By this proposition, if $\widetilde{I}_{\beta}: K_{q}^{\alpha, p} \rightarrow \operatorname{Lip}_{\varepsilon}$ then $\varepsilon=\beta-n / q-\alpha$. Therefore, if $\widetilde{I}_{\beta}: K_{q}^{\alpha, p} \rightarrow \operatorname{Lip}_{\beta-n / q}$ then $\alpha=0$. Furthermore we give the following counterexample on $\mathbb{R}^{1}$.

Counterexample. If $\varepsilon>0$, then $\widetilde{I}_{\beta}$ is not bounded from $K_{q}^{\alpha, p}\left(\mathbb{R}^{1}\right)$ to $\operatorname{Lip}_{\beta-1 / q+\varepsilon}^{-\alpha-\varepsilon}\left(\mathbb{R}^{1}\right)$. 
Since $\operatorname{Lip}_{\beta-1 / q+\varepsilon}^{-\alpha-\varepsilon}\left(\mathbb{R}^{1}\right) \subset \operatorname{Lip}_{\beta-1 / q}^{-\alpha}\left(\mathbb{R}^{1}\right)$ by (4), this counterexample shows that Theorem 2 is optimal.

Proof. Let $f_{j}(x)=2^{-j \alpha} \chi_{\left[2^{j}, 2^{j}+1\right]}(x)$ for $j \geq 1$. Then $\left\|f_{j}\right\|_{K_{q}^{\alpha, p}}=1$. We calculate the mean oscillation of $\widetilde{I}_{\beta} f_{j}$ on intervals $B_{j}=\left(2^{j}-\right.$ $\left.1,2^{j}\right)$ : the center is $2^{j}-1 / 2$ and the radius is $1 / 2$. Since

$$
\widetilde{I}_{\beta} f\left(x_{1}\right)-\widetilde{I}_{\beta} f\left(x_{2}\right)=\int_{\mathbb{R}^{1}}\left\{\frac{1}{\left|x_{1}-y\right|^{1-\beta}}-\frac{1}{\left|x_{2}-y\right|^{1-\beta}}\right\} f(y) d y,
$$

it follows that for $x \in B_{j}$,

$$
\widetilde{I}_{\beta} f_{j}(x)-\widetilde{I}_{\beta} f_{j}\left(2^{j}\right)=\frac{2^{-j \alpha}}{\beta}\left\{\left(2^{j}+1-x\right)^{\beta}-\left(2^{j}-x\right)^{\beta}-1\right\},
$$

and we have

$$
\left(\widetilde{I}_{\beta} f_{j}\right)_{B_{j}}:=\frac{1}{\left|B_{j}\right|} \int_{B_{j}} \widetilde{I}_{\beta} f_{j}(x) d x=\frac{2^{-j \alpha}\left(2^{\beta+1}-\beta-3\right)}{\beta(\beta+1)}+\widetilde{I}_{\beta} f_{j}\left(2^{j}\right) .
$$

Therefore

$$
\begin{aligned}
& \frac{1}{\left|B_{j}\right|} \int_{B_{j}}\left|\widetilde{I}_{\beta} f_{j}(x)-\left(\widetilde{I}_{\beta} f_{j}\right)_{B_{j}}\right| d x \\
& =\frac{2^{-j \alpha}}{\beta} \int_{2^{j}-1}^{2^{j}}\left|\left(2^{j}+1-x\right)^{\beta}-\left(2^{j}-x\right)^{\beta}-1-\frac{2^{\beta+1}-\beta-3}{\beta+1}\right| d x \\
& =\frac{2^{-j \alpha}}{\beta} \int_{0}^{1}\left|(2-x)^{\beta}-(1-x)^{\beta}-1-\frac{2^{\beta+1}-\beta-3}{\beta+1}\right| d x=C_{\beta} 2^{-j \alpha},
\end{aligned}
$$

where $C_{\beta}=\beta^{-1} \int_{0}^{1}\left|(2-x)^{\beta}-(1-x)^{\beta}-1-\left(2^{\beta+1}-\beta-3\right) /(\beta+1)\right| d x \neq 0$. We obtain

$$
\begin{aligned}
& \lim _{j \rightarrow \infty} \frac{1}{\left(\left|2^{j}-1 / 2\right|+1 / 2\right)^{-\alpha-\varepsilon}\left|B_{j}\right|^{1+\beta-1 / q+\varepsilon}} \int_{B_{j}}\left|\widetilde{I}_{\beta} f_{j}(x)-\left(\widetilde{I}_{\beta} f_{j}\right)_{B_{j}}\right| d x \\
& =\lim _{j \rightarrow \infty} C_{\beta} 2^{j \varepsilon}=\infty .
\end{aligned}
$$

\section{References}

[1] A. Beurling, Construction and analysis of some convolution algebras, Ann. Inst. Fourier (Grenoble), 14 (1968), 1-32. 
[2] Eridani, H. Gunawan and E. Nakai, On generalized fractional integral operators, Sci. Math. Jpn., 60 (2004), 539-550.

[3] J. García-Cuerva, Hardy spaces and Beurling algebras, J. London Math. Soc., 39 (1989), 499-513.

[4] E. Hernández and D. Yang, Interpolation of Herz-type Hardy spaces, Illinois J. Math., 420 (1998), 564-581.

[5] C. Herz, Lipschitz spaces and Bernstein's theorem on absolutely convergent Fourier transforms, J. Math. Mech., 18 (1968), 283324.

[6] Y. Komori and K. Matsuoka, Boundedness of several operators on weighted Herz spaces, J. Funct. Spaces Appl., 7 (2009), 112.

[7] X. Li and D. Yang, Boundedness of some sublinear operators on Herz spaces, Illinois J. Math., 40 (1996), 484-501.

[8] S. Z. Lu and D. Yang, Hardy-Littlewood-Sobolev theorems of fractional integration on Herz-type spaces and its applications, Canad. J. Math., 48 (1996), 363-380.

[9] S. Z. Lu and D. Yang, The continuity of commutators on Herztype spaces, Michigan Math. J., 44 (1997), 255-281.

[10] E. Nakai, Pointwise multipliers for functions of weighted bounded mean oscillation, Studia Math., 105 (1993), 105-119.

[11] E. Nakai, On generalized fractional integrals on the weak Orlicz spaces, $B M O_{\phi}$, the Morrey spaces and the Campanato spaces, in Function spaces, interpolation theory and related topics. Proceedings of the international conference in honour of Jaak Peetre on his 65th birthday (Lund, 2000), M. Cwikel et al. (eds.), Walter De Gruyter, (2002), 389-401.

[12] J. Peetre, On the theory of $\mathcal{L}_{p, \lambda}$ spaces, J. Funct. Anal., 4 (1969), 71-87.

[13] L. Tang, Endpoint estimates for multilinear fractional integrals, J. Aust. Math. Soc., 84 (2008), 419-429.

[14] A. Torchinsky, Real-Variable Methods in Harmonic Analysis, Academic Press, 1986.

School of High Technology for Human Welfare, Tokai University 317 Nishino Numazu, Shizuoka 410-0395, Japan

(E-mail : komori@wing.ncc.u-tokai.ac.jp)

(Received : November 2009) 


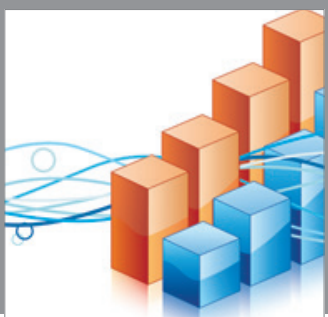

Advances in

Operations Research

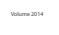

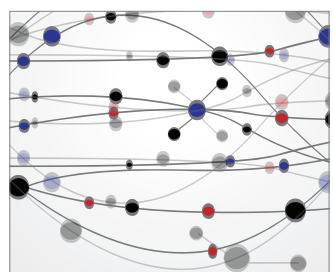

\section{The Scientific} World Journal
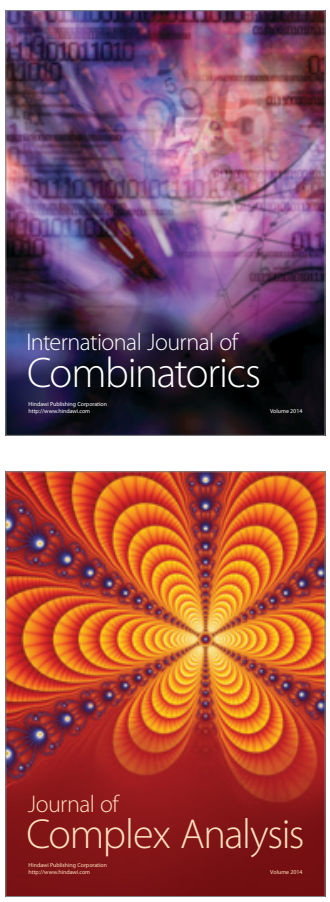

International Journal of

Mathematics and

Mathematical

Sciences
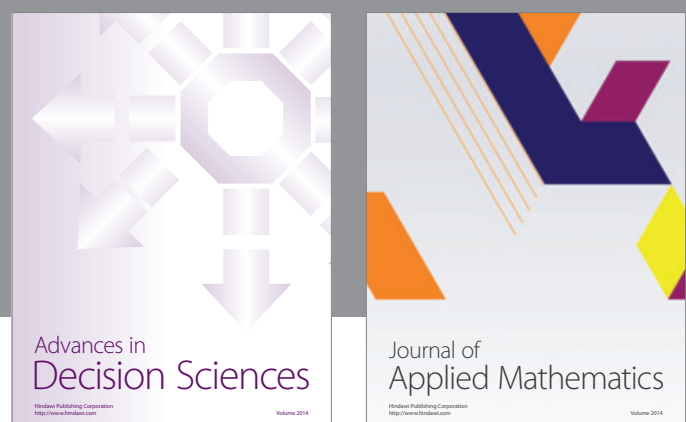

Journal of

Applied Mathematics
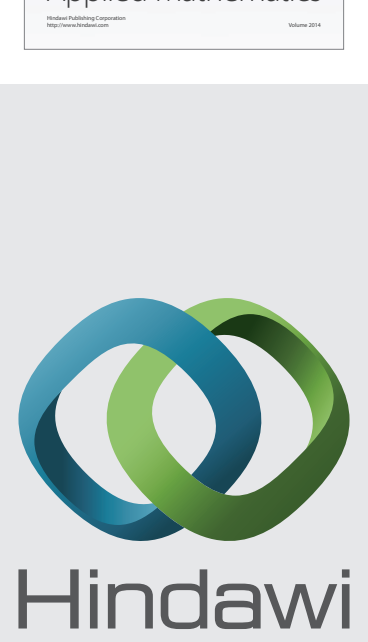

Submit your manuscripts at http://www.hindawi.com
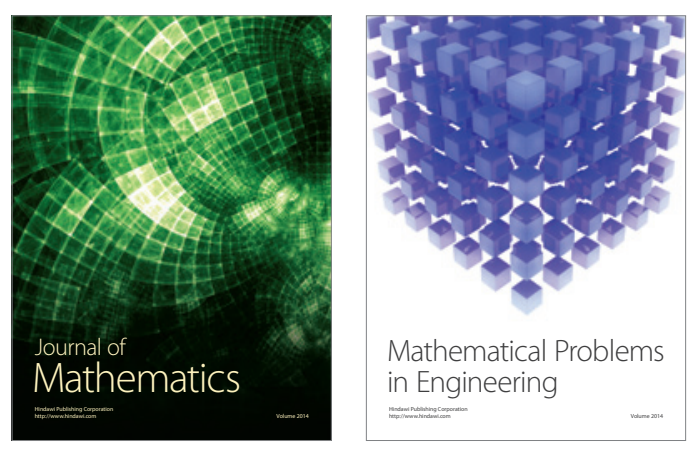

Mathematical Problems in Engineering
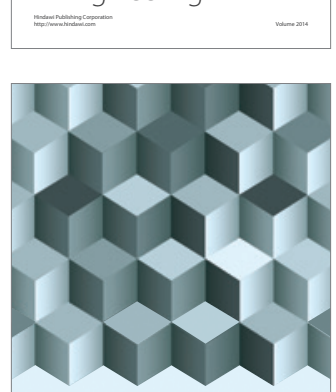

Journal of

Function Spaces
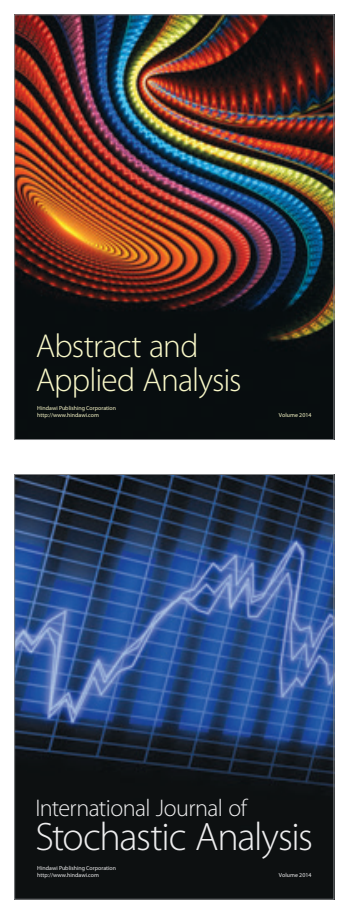

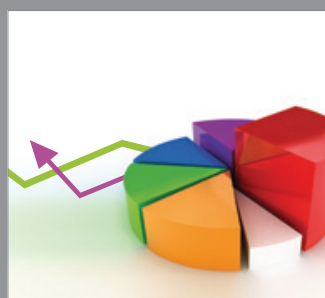

ournal of

Probability and Statistics

Promensencen
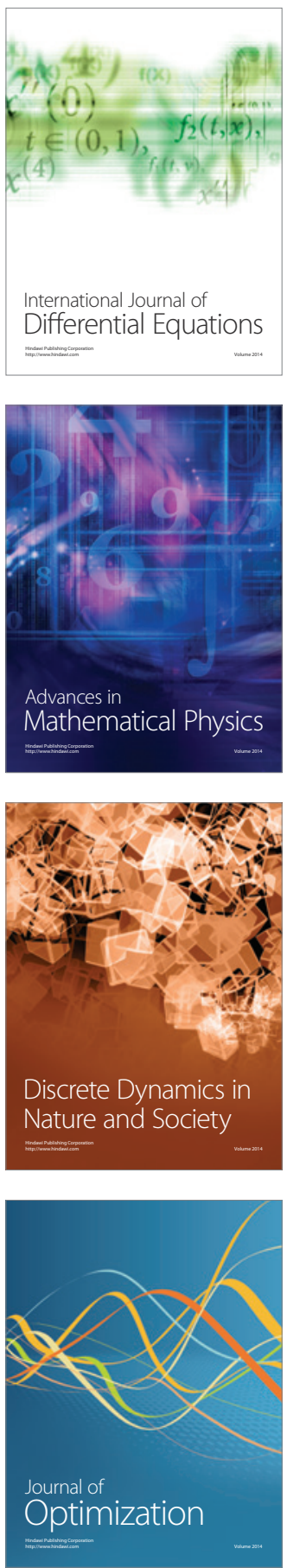